\title{
ABSOLUTE VALUE VARIATIONAL INEQUALITIES AND DYNAMICAL SYSTEMS
}

\author{
SAFEERA BATOOL, MUHAMMAD ASLAM NOOR* AND KHALIDA INAYAT NOOR \\ Department of Mathematics, COMSATS University Islamabad, Islamabad, Pakistan \\ *Corresponding author: noormaslam@gmail.com
}

\begin{abstract}
In this paper, we consider the absolute value variational inequalities. We propose and analyze the projected dynamical system associated with absolute value variational inequalities by using the projection method. We suggest different iterative algorithms for solving absolute value variational inequalities by discretizing the corresponding projected dynamical system. The convergence of the suggested methods is proved under suitable constraints. Numerical examples are given to illustrate the efficiency and implementation of the methods. Results proved in this paper continue to hold for previously known classes of absolute value variational inequalities.
\end{abstract}

\section{INTRODUCTION}

Variational inequalities theory was introduced earlier by Stampacchia [45] and now it is developed as a well-established branch of nonlinear analysis and optimization. Variational inequalities theory is widely applied in industry, economics, social, pure and applied sciences, see [17, 20,27-29,31,32,35-38]. In fact, variational inequalities theory provides us the direct, natural, unified and dynamic framework for the natural analysis of a wide range of unrelated linear and nonlinear problems, see $[5,7,11-13,23]$. Since the discovery of variational inequalities theory, a number of numerical methods including projection method, Wiener-Hopf equations, auxiliary principle and dynamical systems has been developed for solving the variational inequalities and the related optimization problems, see the references therein [1-49].

Received January $29^{\text {th }}, 2020$; accepted February $24^{\text {th }}, 2020$; published May $1^{\text {st }}, 2020$.

2000 Mathematics Subject Classification. 49J40, 65N30, 26D10, 90C23.

Key words and phrases. absolute value variational inequalities; dynamical systems; iterative methods; convergence.

(C)2020 Authors retain the copyrights of their papers, and all open access articles are distributed under the terms of the Creative Commons Attribution License. 
The relationship of variational inequalities and complementarity problems was proved by Karamardian [15], who proved that, if the involved set is a convex cone, then variational inequalities are equivalent to complementarity problem. Complementarity problem was introduced by Lemke [19]. The equivalence between variational inequalities and complementarity problem has been used in suggesting many iterative algorithms for solving complementarity problems, see [17,26,27,37]. Absolute value complementarity problem is an important and useful generalization of the complementarity problem. Absolute value complementarity problem was introduced by Noor et al. [41] and he also shown that absolute value complementarity problem is equivalent to absolute value variational inequalities. Absolute value variational inequalities include the variational inequalities as a special case. It is also proved that if the underlying set is the whole space, then absolute value variational inequalities transform into absolute system of equations, which were introduced and studied by Mangasarian [23], Rohn [43] and Hu and Huang [13]. For the applications of absolute system of equations, see $[13,14,24,25,41,42]$.

Fixed point theory played an important role in developing various types of algorithms for finding the solution of variational inequalities. The equivalence between variational inequalities and fixed point problem can be established by using the projection technique, see [17]. This alternative formulation has been used to suggest the projected dynamical systems related to variational inequalities. Projected dynamical systems were introduced by Dupuis and Nagurney, see [7]. The projected dynamical systems are identified by the discontinuous right hand side. An innovative aspect of the projected dynamical system is that the set of its stationary points corresponds to the solution set of the associated variational inequality problems. Hence the equilibrium problems in different branches of pure and applied sciences which are studied in the framework of variational inequalities, can now be considered in the more general framework of projected dynamical systems. Projected dynamical systems are effective in the development of many efficient numerical techniques for approximating the solutions of variational inequalities and related nonlinear problems, see $[3,6,10,11]$. The global asymptotic stability of the projected dynamical systems has also been studied by Noor [33] and Xia and Wang [46].

In this paper, we consider the absolute value variational inequalities. We propose and analyze the projected dynamical system associated with absolute value variational inequalities by using projection method. It is important to mention that such type of the dynamical systems for absolute value variational inequalities have not been investigated earlier. We suggest different iterative algorithms for the solution of absolute value variational inequalities by dicretizing the corresponding projected dynamical system. The convergence of the suggested methods is proved under suitable conditions. Two examples are given to illustrate the implementation and efficiency of the proposed iterative methods. Since the absolute value 
variational inequalities include the classical variational inequalities, complementarity problems and absolute value equations as special cases, the results obtained in this paper continue to hold for these problems.

\section{Formulations and Basic Results}

Let $H$ be a real Hilbert space, whose norm and inner product are denoted by $\|$.$\| and \langle.,$.$\rangle respectively.$ Let $K$ be a closed and convex set in $H$. For given operators $T, B: H \rightarrow H$, consider the problem of finding $u \in K$ such that

$$
\langle T u+B|u|-f, v-u\rangle \geq 0, \quad \forall v \in K,
$$

where $f$ is a continuous functional defined on $H$ and $|u|$ contains the absolute values of components of $u \in H$. The inequality (1) is called absolute value variational inequality.

We will now discuss some special cases of the problem (1).

(I). If $B|u|=0, \forall u \in H$, then (1) is equivalent to find $u \in K$ such that

$$
\langle T u-f, v-u\rangle \geq 0, \quad \forall v \in K
$$

Inequalities of type equations (2) are known as variational inequalities, which were introduced by Lions and Stampacchia [20] and have been studied extensively in recent years, see [20, 28, 29, 32, 35-38].

(II). If $K^{*}=\{u \in K:\langle u, v\rangle \geq 0, v \in K\}$ is the polar cone of the closed and convex cone $K$ in $H$, then problem (1) is equivalent to find $u \in K$ such that

$$
u \in K, \quad T u+B|u|-f \in K^{*}, \quad\langle T u+B|u|-f, u\rangle=0,
$$

which is called an absolute value complementarity problem. If $B|u|=0$, then problem (3) is known as the complementarity problem, the origin of which can be traced back to Lemke [19] and have been studied by Cottle and Dantzig [4] who restated the linear and quadratic programming problem as a complementarity problem.

(III). If $K=H$, then problem (1) is equivalent to find $u \in H$ such that

$$
T u+B|u|-f=0,
$$

which is called the system of absolute value equations. Absolute system of equations was introduced by Mangasarian and Meyer [24] and was investigated by Hu and Huang [13] in a more general context. A generalized Newton method was also suggested to find the solution of absolute system of equations, see [14]. An algorithm to compute all the solutions of (4) was proposed by Rohn [44] and equivalent formulations of 
(4) are presented by Prokopyev [43]. For the applications of absolute system of equations in various areas of engineering and mathematics, see [11,12,23-25].

Hence, it is clear that the absolute value variational inequality (1) is more general and includes previously known classes of variational inequalities and the system of absolute value equations as special cases. For the recent applications of absolute value variational inequalities, see [40,41].

In order to derive the main results of this paper, we recall some standard definitions and results.

Definition 2.1. An operator $T: H \rightarrow H$ is said to be strongly monotone, if there exists a constant $\alpha>0$ such that

$$
\langle T u-T v, u-v\rangle \geq \alpha\|u-v\|^{2}, \quad \forall u, v \in H
$$

Definition 2.2. An operator $T: H \rightarrow H$ is said to be Lipchitz continuous, if there exists a constant $\beta>0$ such that

$$
\|T u-T v\| \leq \beta\|u-v\|, \quad \forall u, v \in H
$$

If $T$ is strongly monotone and Lipchitz continuous operator, then from definitions (2.1) and (2.2), we have $\alpha \leq \beta$.

Definition 2.3. An operator $T: H \rightarrow H$ is said to be monotone, if

$$
\langle T u-T v, u-v\rangle \geq 0, \quad \forall u, v \in H
$$

Definition 2.4. An operator $T: H \rightarrow H$ is said to be pseudomonotone, if

$$
\langle T u, v-u\rangle \geq 0
$$

implies

$$
\langle T v, v-u\rangle \geq 0 \quad \forall u, v \in H
$$

We now consider the well-known projection lemma [17]. This result is useful to reformulate the variational inequalities as a fixed point problem.

Lemma 2.1. [17] Let $K$ be a closed and convex set in $H$. Then for a given $z \in H, u \in K$ satisfies

$$
\langle u-z, v-u\rangle \geq 0, \quad \forall v \in K
$$


if and only if

$$
u=P_{K} z
$$

where $P_{K}$ is the projection of $H$ onto a closed and convex set $K$ in $H$.

The above lemma plays an important role in obtaining the main results of this paper.

The projection operator $P_{K}$ has following properties:

(1) The projection operator is non-expansive, that is,

$$
\left\|P_{K} u-P_{K} v\right\| \leq\|u-v\|, \quad \forall u, v \in H .
$$

(2) The projection operator $P_{K}$ is co-coercive map, that is,

$$
\left\langle P_{K} u-P_{K} v, u-v\right\rangle \geq\left\|P_{K} u-P_{K} v\right\|^{2}, \quad \forall u, v \in H .
$$

\section{Existence Theory}

In this section, we consider a new type of projected dynamical system associated with absolute value variational inequalities and discuss the existence of the solution, stability and convergence of that dynamical system to the solution of absolute value variational inequality (1). Using Lemma 2.1, we can now state the following result which shows that problem (1) is equivalent to a fixed point problem.

Lemma 3.1. Let $K$ be a closed convex set in $H$. Then $u \in K$ is a solution of absolute value variational inequality (1), if and only if, $u \in K$ satisfies the relation

$$
u=P_{K}[u-\rho T u-\rho B|u|+\rho f],
$$

where $\rho>0$ is a constant.

This alternative equivalent formulation is very important from the theoretical as well as from the numerical point of view. This equivalent provides important tools to approximate the solution of variational inequalities. This equivalent formulation played an important part in establishing the various explicit and implicit iterative methods. Koperlevich [18] developed the the extragradient method for solving the variational inequalities, the convergence of which requires only the Lipschitz continuity.

We now define the residue vector $R(u)$ by the following relation

$$
R(u)=u-P_{K}[u-\rho T u-\rho B|u|+\rho f] .
$$


Using Lemma 3.1, one can conclude that $u \in K$ is a solution of the absolute value variational inequality (1), if and only if, $u \in K$ is a zero of the equation

$$
R(u)=0
$$

Using (7), we suggest a new projected dynamical system related to absolute value variational inequality (1) as follows:

Find $u \in K$ such that

$$
\begin{aligned}
\frac{d u}{d t} & =-\gamma R(u) \\
& =\gamma\left\{P_{K}[u-\rho T u-\rho B|u|+\rho f]-u\right\}, u\left(t_{0}\right)=u_{0} \in K
\end{aligned}
$$

where $\gamma>0$ is a parameter. The expression on the right hand side of (9) is discontinuous on the boundary of $K$. It can be observed from the definition that the solution of (9) always belongs to $K$. Hence, it is meaningful to discuss the existence, uniqueness and continuous dependence of the solution of (9). These type of projected dynamical systems, corresponding to variational inequalities, have been investigated widely, see [3, 6, 7, 9-11,33, 46-48].

The dynamical system is said to be stable in the Lyapunov sense, if the small initial perturbation does not grow in time for a phase trajectory and asymptotically stable, if the small perturbation vanishes as the time goes by. If the dynamical system is asymptotically stable, then it is Lyapunov stable but not conversely, in general.

Definition 3.1. The dynamical system is said to converge globally to the solution set $\widehat{K}$ of (1), if and only if, irrespective of the initial point, the trajectory of the dynamical system satisfies

$$
\lim _{n \rightarrow \infty} \operatorname{dist}(u(t), \widehat{K})=0
$$

where

$$
\operatorname{dist}(u, \widehat{K})=i n f_{v \in \widehat{K}}\|u-v\| \text {. }
$$

It can be observed that if the set $\widehat{K}$ contains a unique point $\widehat{u}$, then (10) implies that

$$
\lim _{n \rightarrow \infty} u(t)=\widehat{u}
$$

Here, the Lyapunov stability of the dynamical system at $\widehat{u}$ implies the global exponential stability of the dynamical system at $\widehat{u}$. 
Definition 3.2. The dynamical system is said to be globally exponentially stable with degree $\lambda>0$, at $\widehat{u} \in \widehat{K}$ if and only if, irrespective of the initial point, the trajectory of the system $u(t)$ satisfies

$$
\|u(t)-\widehat{u}\| \leq \eta_{1}\left\|u\left(t_{0}\right)-\widehat{u}\right\| e^{-\lambda\left(t-t_{0}\right)}, \quad \forall t \geq t_{0},
$$

where $\eta_{1}>0$ and $\lambda>0$, are independent of the initial point. It can also be noted that global exponential stability always implies the global asymptotic stability and hence the dynamical system converges arbitrarily fast.

Lemma 3.2. [34] (Gronwall lemma) Let $u$ and $v$ be real valued non-negative continuous function with domain $\left\{t: t \geq t_{0}\right\}$ and let $\alpha(t)=\alpha_{0}\left|t-t_{0}\right|$, where $\alpha_{0}$ is a monotonically increasing function. If for $t \geq t_{0}$

$$
u(t) \leq \alpha(t)+\int_{t_{0}}^{t} u(x) v(x) d x
$$

then

$$
u(t) \leq \alpha(t) \cdot \exp \left\{\int_{t_{0}}^{t} v(x) d x\right\}
$$

Now onwards, we assume that the nonempty set $\widehat{K}$ is bounded, unless otherwise specified.

We now discuss the existence of the solution of absolute value variational inequality (1) via dynamical system (9), mainly using the technique of Noor [33] and Xia and Wang [46].

Theorem 3.1. Let the operators $T$ and $B$ be Lipchitz continuous with constants $\beta_{1}>0$ and $\beta_{2}>0$, respectively. If $\gamma>0$ and lemma 6 holds, then, for each $u_{0} \in K$, there exists a unique and continuous solution $u(t)$ of the dynamical system (9) with $u\left(t_{0}\right)=u_{0}$ over $\left[t_{0}, \infty\right]$.

Proof. Let

$$
H(u)=\gamma\left\{P_{K}[u-\rho T u-\rho B|u|+\rho f]-u\right\}
$$

where $\gamma>0$ is a constant. For all $u, w \in K$, consider

$$
\begin{aligned}
\|H(u)-H(w)\| \leq & \gamma\{\|u-w\| \\
& \left.+\left\|P_{K}[u-\rho T u-\rho B|u|+\rho f]-P_{K}[w-\rho T w-\rho B|w|+\rho f]\right\|\right\} \\
\leq & \gamma\{\|u-w\|+\|u-w\| \rho\|T u-T w\|+\rho\|B|u|-B|w|\| \\
\leq & \gamma\left\{\|u-w\|+\|u-w\| \rho \beta_{1}\|u-w\|+\rho \beta_{2}\||u|-|w|\|\right. \\
\leq & \gamma\left\{\|u-w\|+\|u-w\| \rho \beta_{1}\|u-w\|+\rho \beta_{2}\|u-w\|\right. \\
\leq & \gamma\left(2+\rho\left(\beta_{1}+\beta_{2}\right)\right)\|u-w\| .
\end{aligned}
$$

where $\beta_{1}>0$ and $\beta_{2}>0$ are Lipchitz constants of the operators $T$ and $B$ respectively. This shows that the operator $H(u)$ is Lipchitz continuous in $K$. So, for each $u_{0} \in K$, there exists a continuous and unique 
solution $u(t)$ of the dynamical system (9), which is defined in an interval $t_{0} \leq t \leq T$ with initial condition $u\left(t_{0}\right)=u_{0}$. Let its maximal interval of existence be $\left[t_{0}, T\right)$. For any $u \in H$, consider

$$
\begin{aligned}
\left\|\frac{d u}{d t}\right\|= & \|H(u)\| \\
= & \gamma\left\{\| P_{K}[u-\rho T u-\rho B|u|+\rho f]-u\right\} \\
\leq & \gamma\left\{\left\|P_{K}[u-\rho T u-\rho B|u|+\rho f]-P_{K}[u]\right\|+\left\|P_{K}[u]-P_{K}[\widehat{u}]\right\|\right. \\
& \left.+\left\|P_{K}[\widehat{u}]-u\right\|\right\} \\
\leq & \gamma\left\{\rho\|T u\|+\rho\|B|u|\|+\|\rho f\|+\|u-\widehat{u}\|+\left\|P_{K}[\widehat{u}]\right\|+\|u\|\right\} \\
\leq & \gamma\left\{\rho \beta_{1}\|u\|+\rho \beta_{2}\|\| u \mid\|+\| \rho f\|+\| u\|+\| \widehat{u}\|+\| P_{K}[\widehat{u}]\|+\| u \|\right\} \\
\leq & \gamma\left(2+\rho\left(\beta_{1}+\beta_{2}\right)\right)\|u\|+\gamma\left\{\left\|P_{K}[\widehat{u}]\right\|+\|\widehat{u}\|+\|\rho f\|\right\},
\end{aligned}
$$

where the Lipchitz continuity of the operators $T$ and $B$ with constants $\beta_{1}>0, \beta_{2}>0$, is used.

Integrating (12) from $t_{0}$ to $t$, we obtain

$$
\begin{aligned}
\|u(t)\| & \leq\left\|u_{0}\right\|+\int_{t_{0}}^{t}\|S u(z)\| d z \\
& \leq\left(\left\|u_{0}\right\|+k_{1}\left(t-t_{0}\right)\right)+k_{2} \int_{t_{0}}^{t}\|u(z)\| d z
\end{aligned}
$$

where,

$$
k_{1}=\gamma\left\{\left\|P_{K}[\widehat{u}]\right\|+\|\widehat{u}\|+\|\rho f\|\right\}
$$

and

$$
k_{2}=\gamma\left(2+\rho\left(\beta_{1}+\beta_{2}\right)\right)
$$

Hence, by using Gronwall's lemma 3.2, we have

$$
\|u(t)\| \leq\left\{\left\|u_{0}\right\|+k_{1}\left(t-t_{0}\right)\right\} e^{k_{2}\left(t-t_{0}\right)}, \quad t \in\left[t_{0}, S\right] .
$$

This proves that the solution $u(t)$ is bounded on the interval $\left[t_{0}, S\right)$. Hence, $S=\infty$.

We now study the stability of dynamical system (9) by using the technique of Noor [33] and Xia and Wang [46].

Theorem 3.2. Let $T$ and $B$ be pseudomonotone and Lipchitz continuous operators with constants $\beta_{1}>$ $0, \beta_{2}>0$, respectively. Then the dynamical system (9) is stable in the Lyapunov sense and converges globally to the solution of the absolute value variational inequality (1).

Proof. Since the operators $T$ and $B$ are Lipchitz continuous with constants $\beta_{1}>0, \beta_{2}>0$. It follows from Theorem 3.1, that the dynamical system (9) has a continuous and unique solution $u(t)$ over the interval 
$\left[t_{0}, S\right)$ for any fixed $u_{0} \in K$. Let $u(t)=u\left(t, t_{0} ; u_{0}\right)$ be the solution of the initial value problem (9). For a given $\widehat{u} \in K$, consider the following Lyapunov function

$$
L(u)=\|u-\widehat{u}\|^{2}, \quad u \in K .
$$

It can be observed that $\lim _{n \longrightarrow \infty} L\left(u_{n}\right)=+\infty$ whenever the sequence $u_{n} \subset K$ and $\lim _{n \rightarrow \infty} u_{n}=+\infty$. Therefore, it can be concluded that the level sets of $L$ are bounded.

Let $\bar{u} \in K$ be a solution of (1). Then

$$
\langle T \widehat{u}+B|\widehat{u}|-f, w-\widehat{u}\rangle \geq 0, \quad \forall w \in K .
$$

Using the pseudomonotonicity of the operators $T$ and $B$ in (14), we obtain

$$
\langle T w+B|w|-f, w-\widehat{u}\rangle \geq 0 .
$$

Taking $w=P_{K}[u-\rho T u-\rho B|u|+\rho f]$ in (15), we have

$$
\left\langle T P_{K}[u-\rho T u-\rho B|u|+\rho f], P_{K}[u-\rho T u-\rho B|u|+\rho f]-\widehat{u}\right\rangle \geq 0 .
$$

Setting

$$
\begin{gathered}
v=\widehat{u}, \\
u=P_{K}[u-\rho T u-\rho B|u|+\rho f],
\end{gathered}
$$

and

$$
z=u-\rho T P_{K}[u-\rho T u-\rho B|u|+\rho f]
$$

in (6), we get

$$
\begin{aligned}
\left\langle P_{K}[u-\rho T u-\rho B|u|+\right. & \rho f]-u+\rho T P_{K}[u-\rho T u-\rho B|u|+\rho f], \widehat{u}-P_{K}[u] \\
& -\rho T u-\rho B|u|+\rho f]\rangle \geq 0 .
\end{aligned}
$$

Adding (16) and (17)

$$
\begin{array}{r}
\left\langle-\rho T P_{K}[u-\rho T u-\rho B|u|+\rho f]+P_{K}[u-\rho T u-\rho B|u|+\rho f]-u+\right. \\
\left.\rho T P_{K}[u-\rho T u-\rho B|u|+\rho f], \widehat{u}-P_{K}[u]\right\rangle \geq 0,
\end{array}
$$

and using (8), we get

$$
\langle-R(u), \widehat{u}-u+R(u)\rangle \geq 0
$$

which shows that

$$
\langle u-\widehat{u}, R(u)\rangle \geq\|R(u)\|^{2} .
$$


Hence from (9), (13) and (19), we get

$$
\begin{aligned}
\frac{d}{d t} L(u) & =\frac{d L}{d u} \frac{d u}{d t} \\
& =\left\langle 2(u-\widehat{u}), \gamma\left\{P_{K}[u-\rho T u-\rho B|u|+\rho f]-u\right\}\right\rangle \\
& =2 \gamma\langle u-\widehat{u}, R(u)\rangle \\
& \leq-2 \gamma\|R(u)\|^{2} \leq 0 .
\end{aligned}
$$

This proves that $L(u)$ is global Lyapunov function for the dynamical system (9) and hence the dynamical system (9) is Lyapunov stable. Since, we have

$\left\{u(t): t \geq t_{0}\right\} \subset K_{0}$, where $K_{0}=\left\{u \in K: L(u) \leq L\left(u_{0}\right)\right\}$ and $L(u)$ is continuously differentiable function on the closed and bounded set $K$, LaSalle's invariance principle shows the convergence of the trajectory to the largest subset $\varphi$ of the following subset:

$$
X=\left\{u \in K ; \frac{d L}{d t}=0\right\} .
$$

If

$$
\frac{d L}{d t}=0
$$

then, from (20), we have

$$
\|R(u)\|^{2}=0
$$

that is

$$
\left\|u-P_{K}[u-\rho T u-\rho B|u|+\rho f]\right\|=0 .
$$

Using (21) in (9), we get

$$
\frac{d u}{d t}=0,
$$

which implies that $u$ is an equilibrium point of the system (9).

Conversely, if $\frac{d u}{d t}=0$. Then from(20), we have

$$
\frac{d L}{d t}=0
$$

Thus, we conclude that

$$
X=\left\{u \in K ; \frac{d L}{d t}=0\right\}=K_{0} \cap \widehat{K},
$$

where the nonempty set $X$ is convex and invariant set which is contained in the solution set $\widehat{K}$. So,

$$
\lim _{n \rightarrow \infty} \operatorname{dis}(u(t), X)=0 .
$$

Using definition 7 and (22), we obtain the global convergence of the dynamical system (9) to the solution set of (1). In particular, if $X=\{\widehat{u}\}$, then

$$
\lim _{n \rightarrow \infty} u(t)=\widehat{u}
$$


which proves that the dynamical system (9) is globally asymptotically stable.

Theorem 3.3. Let the operators $T$ and $B$ be Lipchitz continuous with constants $\beta_{1}>0$ and $\beta_{2}>0$, respectively. If $\gamma<0$, then the projected dynamical system (9) globally exponentially converges to the unique solution of the absolute value variational inequality (1).

Proof. It can be seen from Theorem 3.1 that a unique and continuously differentiable solution of the dynamical system (9) exists over the interval $\left[t_{0}, \infty\right)$. So,

$$
\begin{aligned}
\frac{d L}{d t} & =2 \gamma\left\langle u(t)-\widehat{u}, P_{K}[u(t)-\rho T u(t)-\rho B|u(t)|+\rho f]-u(t)\right\rangle \\
& =-2 \gamma\|u(t)-\widehat{u}\|^{2}+2 \gamma\left\langle u(t)-\widehat{u}, P_{K}[u(t)-\rho T u(t)-\rho B|u(t)|+\rho f]-\widehat{u}\right\rangle,
\end{aligned}
$$

where $\widehat{u} \in K$ is the solution of the absolute value variational inequality (1). So, we have

$$
\widehat{u}=P_{K}[\widehat{u}-\rho T \widehat{u}-\rho B|\widehat{u}|+\rho f] .
$$

From the nonexpansivity of $P_{K}$ and the Lipchitz continuity of the operators $T$ and $B$, we have

$$
\begin{aligned}
\| P_{K}[u & -\rho T u-\rho B|u|+\rho f]-P_{K}[\widehat{u}-\rho T \widehat{u}-\rho B|\widehat{u}|+\rho f] \| \\
& \leq\|u-\widehat{u}\|+\rho\|T u-T \widehat{u}\|+\rho\|B|u|-B|\widehat{u}|\| \\
& \leq\left(1+\rho\left(\beta_{1}+\beta_{2}\right)\right)\|u-\widehat{u}\| .
\end{aligned}
$$

From (23) and (24), we obtain

$$
\begin{aligned}
\frac{d}{d t}\|u-\widehat{u}\|^{2} & \leq-2 \gamma\|u(t)-\widehat{u}\|^{2}+2 \gamma\left(1+\rho\left(\beta_{1}+\beta_{2}\right)\right)\|u-\widehat{u}\|^{2} \\
& =-2 \gamma \theta\|u-\widehat{u}\|^{2} .
\end{aligned}
$$

where

$$
\theta=1+\rho\left(\beta_{1}+\beta_{2}\right)
$$

Thus, for $\gamma=-\gamma_{1}$, where $\gamma_{1}$ is a positive constant, we get

$$
\|u-\widehat{u}\|^{2} \leq\left\|u\left(t_{0}\right)-u\right\| e^{-\theta \gamma_{1}\left(t-t_{0}\right)} .
$$

Hence, using definition 8, it is proved that the trajectory of the solution of the system (9) will converge globally exponentially to the unique solution of the absolute value variational inequality (1). 


\section{Iterative Methods}

In this section, we use the projected dynamical system (9) associated with absolute variational inequality (1) to suggest and analyze some iterative schemes, which will be used to obtain the solution of absolute value variational inequalities. For the numerical computations of the suggested algorithms, the projection operator, $P_{K}$, is defined by

$$
P_{K}\left(u_{i}\right)= \begin{cases}u, & \|u-s\| \leq r \\ s+\frac{r(u-s)}{\|u-s\|}, & \|u-s\|>r,\end{cases}
$$

if $K=\left\{u \in R^{n}:\|u-s\| \leq r, s \in R^{n}, r>0\right\}$.

Consider the dynamical system (9) with $\gamma=1$,

$$
\frac{d u}{d t}+u=P_{K}[u-\rho T u-\rho B|u|+\rho f], u\left(t_{0}\right)=u_{0} .
$$

For a given $\eta \in[0,1]$, using forward difference schemes, we discretize (24) and suggest the following iterative algorithms to find the solution of absolute value variational inequalities.

Algorithm 4.1. For a given $u_{0} \in K$, compute $u_{n+1}$ by the iterative scheme

$$
\begin{aligned}
u_{n+1}= & P_{K}\left[\eta\left(u_{n+1}-u_{n}\right)+\frac{1+h}{h} u_{n}-\frac{u_{n+1}}{h}\right. \\
& \left.-\rho T u_{n+1}-\rho B\left|u_{n+1}\right|+\rho f\right], \quad n=0,1,2, \ldots
\end{aligned}
$$

which is an implicit method.

For the implementation of Algorithm 4.1, we suggest the following two-step, iterative method.

Algorithm 4.2. For a given $u_{0} \in K$, compute $u_{n+1}$ by the iterative schemes

$$
\begin{aligned}
w_{n} & =P_{K}\left[u_{n}-\rho T u_{n}-\rho B\left|u_{n}\right|+\rho f\right] \\
u_{n+1} & =P_{K}\left[\eta\left(w_{n}-u_{n}\right)+\frac{1+h}{h} u_{n}-\rho T w_{n}-\rho B\left|w_{n}\right|+\rho f-\frac{w_{n}}{h}\right] .
\end{aligned}
$$

Algorithm 4.2 is a new two-step iterative method for finding the solution of absolute value variational inequality (1).

We also consider some special cases of Algorithm 4.2.

(1) For $\eta=0$, Algorithm 4.2 collapses to the following iterative method. 
Algorithm 4.3. For a given $u_{0} \in K$, compute $u_{n+1}$ by the iterative scheme

$$
\begin{aligned}
w_{n} & =P_{K}\left[u_{n}-\rho T u_{n}-\rho B\left|u_{n}\right|+\rho f\right] \\
u_{n+1} & =P_{K}\left[\frac{1+h}{h} u_{n}-\frac{w_{n}}{h}-\rho T w_{n}-\rho B\left|w_{n}\right|+\rho f\right],
\end{aligned}
$$

Algorithm 4.3 is extragradient type methods in the sense of Korpelevich [18].

(2) For $\eta=1$, Algorithm 4.2 reduces to the following iterative method.

Algorithm 4.4. For a given $u_{0} \in K$, compute $u_{n+1}$ by the iterative scheme

$$
\begin{gathered}
w_{n}=P_{K}\left[u_{n}-\rho T u_{n}-\rho B\left|u_{n}\right|+\rho f\right] \\
u_{n+1}=P_{K}\left[w_{n}-\rho T w_{n}-\rho B\left|w_{n}\right|+\rho f\right] .
\end{gathered}
$$

Algorithm 4.4 is modified extragradient type iterative method in the sense of Noor $[35,36]$.

(3) For $\eta=\frac{1}{2}$, Algorithm 4.2 transforms into the following iterative method.

Algorithm 4.5. For a given $u_{0} \in K$, compute $u_{n+1}$ by the iterative scheme

$$
\begin{aligned}
w_{n} & =P_{K}\left[u_{n}-\rho T u_{n}-\rho B\left|u_{n}\right|+\rho f\right] \\
u_{n+1} & =P_{K}\left[\frac{h+2}{2 h} u_{n}-\frac{h-2}{2 h} w_{n}-\rho T w_{n}-\rho B\left|w_{n}\right|+\rho f\right] .
\end{aligned}
$$

Clearly, Algorithm 4.2 contains the known iterative methods such as extragradient method by Korpelevich [18], modified extragradient method by Noor [35,36] as special cases. This shows that Algorithm 4.2 is quite general and unifying one.

We now discuss the convergence of Algorithm 4.2 which is the main motivation of the next result.

Theorem 4.1. Let $u \in K$ be a solution of absolute value variational inequality (1). Let $u_{n+1}$ be the approximate solution obtained from (26). If $T$ and $B$ are monotone operators, then

$$
\left\|u-u_{n+1}\right\|^{2} \leq\left\|u-u_{n}\right\|^{2}-\left\|u_{n}-u_{n+1}\right\|^{2} .
$$

Proof. Let $u \in K$ be a solution of absolute value variational inequality (1) i.e;

$$
<\langle u+B|u|-f, v-u\rangle \geq 0, \forall v \in K
$$


Using monotonicity of the operators $T$ and $B$, we get

$$
\langle T v+B|v|-f, v-u\rangle \geq 0, \forall v \in K .
$$

Taking $v=u_{n+1}$ in (28), we obtain

$$
\left\langle T u_{n+1}-B\left|u_{n+1}\right|-f, u_{n+1}-u\right\rangle \geq 0 .
$$

Using lemma 2.1, Algorithm 4.1 can be rewritten in the following equivalent form, that is

$$
\begin{aligned}
\left\langle\left(\rho T u_{n+1}+\rho B\left|u_{n+1}\right|-\rho f+\right.\right. & \left.\frac{1+h}{h}-\eta\right) u_{n+1} \\
& \left.\quad-\left(\frac{1+h}{h}-\eta\right) u_{n}, v-u_{n+1}\right\rangle \geq 0 .
\end{aligned}
$$

Subtituting $\mathrm{v}=\mathrm{u}$ in (30) to have,

$$
\begin{aligned}
\left\langle\left(\rho T u_{n+1}+\rho B\left|u_{n+1}\right|-\right.\right. & \rho f+\left(\frac{1+h}{h}-\eta\right) u_{n+1} \\
& \left.-\left(\frac{1+h}{h}-\eta\right) u_{n}, u-u_{n+1}\right\rangle \geq 0 .
\end{aligned}
$$

From (29) and (31), we obtain

$$
\begin{aligned}
\left(\frac{1+h}{h}-\eta\right)\left\langle u_{n+1}-u_{n}, u-u_{n+1}\right\rangle & \geq \rho\left\langle T u_{n+1}+\left|u_{n+1}\right|-f, u_{n+1}-u\right\rangle \\
& \geq 0
\end{aligned}
$$

From (32) and using inequality

$$
2\langle u, v\rangle=\|u+v\|^{2}-\|u\|^{2}-\|v\|^{2}, \quad \forall u, v \in H,
$$

we get

$$
\left\|u-u_{n+1}\right\|^{2} \leq\left\|u-u_{n}\right\|^{2}-\left\|u_{n}-u_{n+1}\right\|^{2},
$$

which is (27), the required result.

Theorem 4.2. Let $u \in K$ be the solution of absolute value variational inequality (1). Let $u_{n+1}$ be the approximate solution obtained from Algorithm 4.1. If $T$ and $B$ are monotone operators, then $u_{n+1}$ converges to $u \in K$ satisfying (1).

Proof. Let $T$ and $B$ be a monotone operators. Then, from (27), it follows that sequence $\left\{u_{i}\right\}_{i=1}^{\infty}$ is a bounded sequence and

$$
\sum_{n=1}^{\infty}\left\|u_{n+1}-u\right\|^{2} \leq\left\|u-u_{0}\right\|^{2}
$$

which shows that

$$
\lim _{n \rightarrow \infty}\left\|u_{n+1}-u_{n}\right\|^{2}=0
$$


Since $\left\{u_{j}\right\}_{j=1}^{\infty}$ is bounded, so there exists a limit point $\bar{u} \in K$ such that subsequence $\left\{u_{j_{l}}\right\}_{l=j}^{\infty}$ converges to. Replacing $u_{n}$ by $u_{n_{j}}$ in (32) and taking the limit $u_{i} \longrightarrow \infty$, we obtain

$$
\langle T \bar{u}+B|\bar{u}|-f, v-\bar{u}\rangle \geq 0, \quad \forall v \in K
$$

which implies that $\bar{u}$ is the solution of (1)and

$$
\left\|u_{n+1}-u\right\|^{2} \leq\left\|u-u_{n}\right\|^{2}
$$

It follows from the above inequality that the limit point $u_{n}$ is unique and

$$
\lim _{n \rightarrow \infty} u_{n+1}=\bar{u}
$$

which completes the proof.

\section{Computational Results}

In this section, we consider two numerical examples to examine the efficiency and implementation of the suggested algorithm 4.2 from the aspects of the number of iterations and the elapsed CPU time in seconds (denoted by TOC). The experiments in both the examples are performed with Intel(i7) 2.2GHz, 8GB RAM, and the codes are written in Matlab R2010.

Example 5.1. [8] Consider random matrix $T$ and $f$, for absolute value variational inequality (1) in Matlab code as

$$
\begin{aligned}
n & =\text { input }(\text { dimensionofmatrix } T=) ; \operatorname{rand}(\text { state }, 0) \\
R & =\operatorname{rand}(n, n) \\
f & =\operatorname{rand}(n, 1) \\
T & =R^{\prime} * R+n * \operatorname{eye}(n) \\
B & =\operatorname{eye}(n)
\end{aligned}
$$

with random initial guess.

The comparison between Algorithm 16, the Yong method [49] and Algorithm 2.1 [8] is presented in Table 1. The average times taken by CPU for every order of $n$ are presented by TOC, in Table 1 . Note that for any size of dimension $n$, Algorithm 16 converges faster than both the Yong method [49] and Algorithm 2.1 [8]. 
TABLE 1. Numerical results for Example 5.1.

\begin{tabular}{|c|c|c|c|c|c|c|}
\hline \multirow{2}{*}{$\begin{array}{c}\text { Order } \\
\text { heading }\end{array}$} & \multicolumn{2}{|c|}{ Yong method } & \multicolumn{2}{|c|}{ Algorithm 2.1 [8] } & \multicolumn{2}{|c|}{ Algorithm 4.2} \\
\hline & No. of iter. & $T O C$ & No. of iter. & TOC & No. of iter. & $T O C$ \\
\hline 4 & 2 & 2.230 & 3 & .00050 & 3 & .001 \\
\hline 8 & 2 & 3.340 & 3 & .00065 & 3 & .00099 \\
\hline 16 & 3 & 3.790 & 3 & .00078 & 3 & .00099 \\
\hline 32 & 2 & 4.120 & 3 & .00101 & 3 & .00099 \\
\hline 64 & 3 & 6.690 & 3 & .01224 & 3 & .001 \\
\hline 128 & 3 & 12.450 & 3 & .04209 & 3 & .001 \\
\hline 256 & 3 & 34.670 & 3 & .06714 & 3 & .00099 \\
\hline 512 & 5 & 79.570 & 3 & .32896 & 3 & .00099 \\
\hline 1024 & 5 & $15 \% .12$ & 3 & .83559 & 3 & .004 \\
\hline
\end{tabular}

Example 5.2. [22] We randomly select a matrix A according to the following structure:

$$
\begin{aligned}
A & =\operatorname{round}(100 *(\operatorname{eye}(n ; n)-.02 *(2 * \operatorname{rand}(n ; n)-1)) ; \\
B & =\operatorname{eye}(n) ; \\
b(i) & =(-i)^{i}, i=1,2, \ldots, n .
\end{aligned}
$$

TABLE 2. Numerical results for Example 5.2.

\begin{tabular}{cccccc}
\hline Order & & $P-S S O R$ & $P-C G$ & $P-J A C O B I$ & Algorithm 4.2 \\
\hline 500 & linter number & 6 & 6 & 18 & 18 \\
& CPU time & .0205 & .0397 & .0040 & .0069 \\
1000 & linter number & 6 & 6 & 18 & 38 \\
& CPU time & .0954 & .1953 & .0573 & .0560 \\
1500 & linter number & 6 & 6 & 31 & 38 \\
& CPU time & .2427 & .4852 & .1613 & .1230 \\
2000 & linter number & 6 & 6 & 38 & 38 \\
& CPU time & .4888 & .9111 & .3541 & .2100
\end{tabular}

From Table 2, all the iteration steps (linter number) and the elapsed CPU times show that Algorithm 16 is more efficient than the P-SSOR, P-JACOBI and P-CG iteration methods. 


\section{ConClusion}

In this paper, we have considered and analyzed dynamical system associate with absolute value variational inequalities. We have discussed the existence of a solution of the absolute value variational inequalities using only the Lipschitz continuity of the underlying operators. Asymptotic stability of the solution is investigated using the Lyapunov functions. Dynamical system is used to consider some extrgradient type methods for solving the absolute value equations. Some numerical examples are given, which shows that the proposed methods perform better then the previous ones. Ideas and techniques of this paper may inspire further research in this dynamical field.

Acknowledgement: The author would like to thank the Rector, COMSATS University Islamabad, Islamabad, Pakistan, for providing excellent research and academic environments

Conflicts of Interest: The author(s) declare that there are no conflicts of interest regarding the publication of this paper.

\section{REFERENCES}

[1] B. H. Ahn, Iterative methods for linear complementarity problems with upper bounds on primary variables, Math. Program. 26(3)(1983), 295-315.

[2] C. Bakxchi and A. Capello, Disequazioni variationalies quasi varionali, applicantioni a problemi di frontiera libera, Vols. I and II, Bologna, Italia. (1978).

[3] B. B. Bin-Mohsin, M. A. Noor, K. I. Noor and R. Latif, Projected dynamical system for variational inequalities, J. Adv. Math. Stud. 11(1)(2018), 1-9.

[4] R. W. Cottle and G. B. Dantzig, Complementarity pivot theory of mathematical programming. Linear Algebra Appl. $1(1968), 103-125$.

[5] I. C. Dolcetta and U. Mosco, Implicit complementarity problems and quasi variational inequalities. Var. Ineq. and Compl. Prob. Th. App. (Eds. R.W. Cottle, F. Giannessi and J.L. Lions) John Wiley and Sons, New York, New Jersey, 1980.

[6] J. Dong, D. H. Zhang and A. Nagurney, A projected dynamical systems model of general financial equilibrium with stability analysis. Math. Comput. Model. 24(2)(1996), 35-44.

[7] P. Dupuis and A. Nagurney, Dynamical systems and variational inequalities. Ann. Oper. Res. 44(1)(1993), 7-42.

[8] H. Esmaeili, M. Mirzapour and E. Mahmoodabadi, A fast convergent two-step iterative method to solve the absolute value equation. U.P.B. Sci. Bull., Ser. A. 78(1)(2016), 25-32.

[9] G. Fichera, G. Problemi elastostatistic con vincoli unilaterali il prolema di signorini con ambigue condizone as contorno atti. Acad. Naz. Lincei. Mem. Cl. Sci. Fis. Mat. Nature. Sez. La., 8(7)(1964), 91-140.

[10] T. L. Friesz, D. H. Berstein, N. J. Mehta, R. L. Tobin and S. Ganjliazadeh, Day-to day dynamic network disequilibrium and idealized traveler information systems. Oper. Res. 42(6)(1994), 1120-1136.

[11] T. L. Friesz, D. H. Berstein and R. Stough, Dynamic systems, variational inequalities and control theoretic models for predicting time-varying urban network flows. Transport. Sci. 30(1)(1996), 14-31.

[12] G. Glowinski, J. L.Lions and R. Tremolieres, Numerical Analysis of variational Inequalities. NorthHolland, Amsterdam, 1981. 
[13] S. L. Hu and Z. H. Huang, A note on absolute value equations. Optim. Lett. 4(3)(2010), $417-424$.

[14] S. L. Hu and Z. H. Huang, A generalized Newton method for absolute value equations associated with second order cones. J. Comput. Appl. Math. 235(5)(2011), 1490-1501.

[15] S. Karamardian, The complementarity problem. Math. Program. 2(1)(1972), 107-109.

[16] Y. F. Ke and C. F. Ma, SOR-like iteration method for solving absolute value equations. Appl. Math. Comput. 311(2017), $195-202$.

[17] D. Kinderlehrer and G. Stampacchia, An Introduction to Variational Inequalities and Their Applications. SIAM, Philadelphia, 1980.

[18] G. M. Korpelevich, An extragradient method for finding saddle points and for other problems. Ekonomika Mat. Metody, $12(4)(1976), 747-756$.

[19] C. E. Lemke, Bimatrix equilibrium points and mathematical programming. Manage. Sci. 11(7)(1965), 681-689.

[20] J. L. Lions and G. Stampacchia, Variational inequalities. Commun. Pure Appl. Math. 20(3)(1967), 493-519.

[21] J. L. Lions, Optimal Control of Systems Governed by Partial Differential Equations. Springer-Verlag, Berlin. 1971.

[22] C. Q. LV and C. F. Ma, Picard splitting method and Picard CG method for solving the absolute value equation. J. Nonlinear Sci. Appl. 10(2017), 3643-3654.

[23] O. L. Mangasarian, The linear complementarity problem as a separable bilinear program. J. Glob. Optim. 6(2)(1995), 153-161.

[24] O. L. Mangasarian and R. R. Meyer, Absolute value equations, Linear Algebra Appl. 419(2-3)(2006), 359-367.

[25] O. L. Mangasarian, Absolute value programming. Addison-Wesley Publishing, Boston. 2007.

[26] K. G. Murty, Linear complementarity, linear and nonlinear programming. Heldermann Verlag, Berlin. 1988.

[27] M. A. Noor, On variational inequalities. Ph.D. thesis. Burnel University, U.K. (1975).

[28] M. A. Noor, Mildly Nonlinear variational inequalities. Mathematica. 24(47)(1982), 99-110.

[29] M. A. Noor, Strongly nonlinear variational inequalities. C. R. Math. Rep. Acad. Sci. Canada. 4(4)(1982), 213-218.

[30] M. A. Noor, Iterative Methods for a Class of Complementarity Problems. J. Math. Anal. Appl. 133(2)(1988), 366-382.

[31] M. A. Noor, Some recent advances in variational inequalities. Part I. Basic concepts. New Zealand J. Math. 26(1)(1997), $53-80$.

[32] M. A. Noor, Some recent advances in variational inequalties. Part II. Other concepts. New Zealand J. Math. 26(2)(1977), $229-255$

[33] M. A. Noor, Stability of the modified projected dynamical systems. Computer Math. Appl. 44(2002), 1-5.

[34] M. A. Noor, Resolvent dynamical systems for mixed variational inequalities. Korean J. Comput. Appl. Math. 9(1)(2002), $15-26$.

[35] M. A. Noor, Some developments in general variational inequalities. Appl. Math. Comput. 152(2004), $199-277$.

[36] M. A. Noor, On an implicit method for nonconvex variational inequalities,.J. Optim. Theory Appl. 147(2)(2010), 411-417.

[37] M. A. Noor and K. I. Noor, Iterative methods for variational inequalities and nonlinear programming. Oper. Res. Verf. 31(1979), 455-463.

[38] M. A. Noor, Y. J. Wang and N. Xiu, Some new projection methods for variational inequalities. Appl. Math. Comput. $137(2)(2003), 423-435$.

[39] M. A. Noor, K. I. Noor and A. Bnouhachem, On a unified implicit method for variational inequalities. J. Comput. Appl. Math. 249(2013), 69-73.

[40] M. A. Noor, M, K. I. Noor and S. Batool, On generalized absolute value equations. U.P.B. Sci. Bull., Series A, 80(4)(2018), 63-70. 
[41] M. A. Noor, J. Iqbal, K. I. Noor and E. Al-Said, Generalized AOR method for solving absolute value complementarity problems J. Appl. Math. 2012(2012), 743861.

[42] O. Prokopyev, O. On equivalent reformulation for absolute value equations. Comput. Optim. App., 44(3)(2009), 363-372.

[43] J. Rohn, A theorem of the alternatives for the equation $A x+B|x|=b$. Linear and Multilinear Algebra, 52(6)(2004), $421-426$

[44] J. Rohn, An algorithm for computing all solutions of an absolute value equation. Optim. Lett. 6(5)(2011), 851-856.

[45] G. Stampacchia, Formes bilineaires coercivites sur les ensembles convexes. C. R. Acad. Sci. Paris. 258(1964), 4413-4416.

[46] Y. S. Xia and J. Wang, On the stability of globally projected dynamical systems. J. Optim. Theory Appl. 106(1)(2000), 129-150.

[47] Y. S. Xia and J. Wang, A recurrent neural network for solving linear projection equation. Neural Networks 13(3)(2000), 337-350.

[48] Y. S. Xia, On convergence conditions of an extended projection neural network. Neural Comput. 17(3)(2005), 515-525.

[49] L. Yong, Particle Swarm Optimization for absolute value equations. J. Comput. Inform. Syst. 6(7)(2010), $2359-2366$. 